\title{
Supplementary Motor Area Syndrome and Flexor Synergy of the Lower Extremities
}

\author{
Ju Seok Ryu, MD ${ }^{1}$, Min Ho Chun, $\mathrm{MD}^{2}$, Dae Sang You, $\mathrm{MD}^{2}$
}

\author{
${ }^{1}$ Department of Rehabilitation Medicine, CHA Bundang Medical Center, CHA University, Seongnam; \\ ${ }^{2}$ Department of Rehabilitation Medicine, Asan Medical Center, University of Ulsan Collage of Medicine, Seoul, Korea
}

\begin{abstract}
Clinical presentation of supplementary motor area (SMA) syndrome includes complete akinesia of the contralateral side of the body and mutism, with secondary recovery of neurologic deficit. Multi-joint coordination is frequently impaired following the development of a brain lesion and is generally restricted by abnormal patterns of muscle activation within the hemiparetic limb, clinically termed muscle synergies. However, no work to date has confirmed this observation with the aid of objective methods, such as gait analysis, and the development of reflex pattern has not been suggested as a possible cause. We describe two unusual cases of flexor synergy after tumor resection of SMA lesions.
\end{abstract}

Keywords Motor cortex, Synergy, Gait, Brain neoplasms

\section{INTRODUCTION}

Clinical presentation of supplementary motor area (SMA) syndrome includes complete akinesia of the contralateral side of the body and mutism, with secondary recovery of neurologic deficit. SMA has been recognized by Penfield and Welch [1] as the area from which synergic movements may be produced.

Multi-joint coordination is frequently impaired following the development of brain lesions and is generally restricted by abnormal patterns of muscle activation within

Received August 7, 2012; Accepted January 18, 2013

Corresponding author: Min Ho Chun

Department of Rehabilitation Medicine, Asan Medical Center, Ulsan University College of Medicine, 88 Olympic-ro 43-gil, Songpa-gu, Seoul 138-736, Korea

Tel: +82-2-3010-3800, Fax: +82-2-3010-6964, E-mail: mhchun@amc. seoul.kr

() This is an open-access article distributed under the terms of the Creative Commons Attribution Non-Commercial License (http://creativecommons. org/licenses/by-nc/3.0) which permits unrestricted noncommercial use, distribution, and reproduction in any medium, provided the original work is properly cited.

Copyright $\odot 2013$ by Korean Academy of Rehabilitation Medicine hemiparetic limb, clinically termed muscle synergies. In the upper extremities, it generally involves a coupling of elbow flexion with shoulder abduction-extensionexternal rotation and to a lesser extent, association of elbow extension with shoulder adduction-flexion-internal rotation $[2,3]$. Studies evaluating the lower extremities of healthy persons have shown that knee flexor and ankle plantar flexor are activated simultaneously, and that knee extensor and ankle dorsiflexor show a similar pattern of activation [4]. Significant differences, however, were observed among activation patterns exhibited by hemiparetic, nonparetic, and normal limbs [2].

Several studies have reported dissociation between the volitional and automatic movements of affected limbs in patients with SMA syndrome. However, no work to date has confirmed this observation with the aid of objective methods such as gait analysis, and development of reflex pattern has not been suggested as a possible cause. We describe two unusual cases of flexor synergy after tumor resection of SMA lesions. 

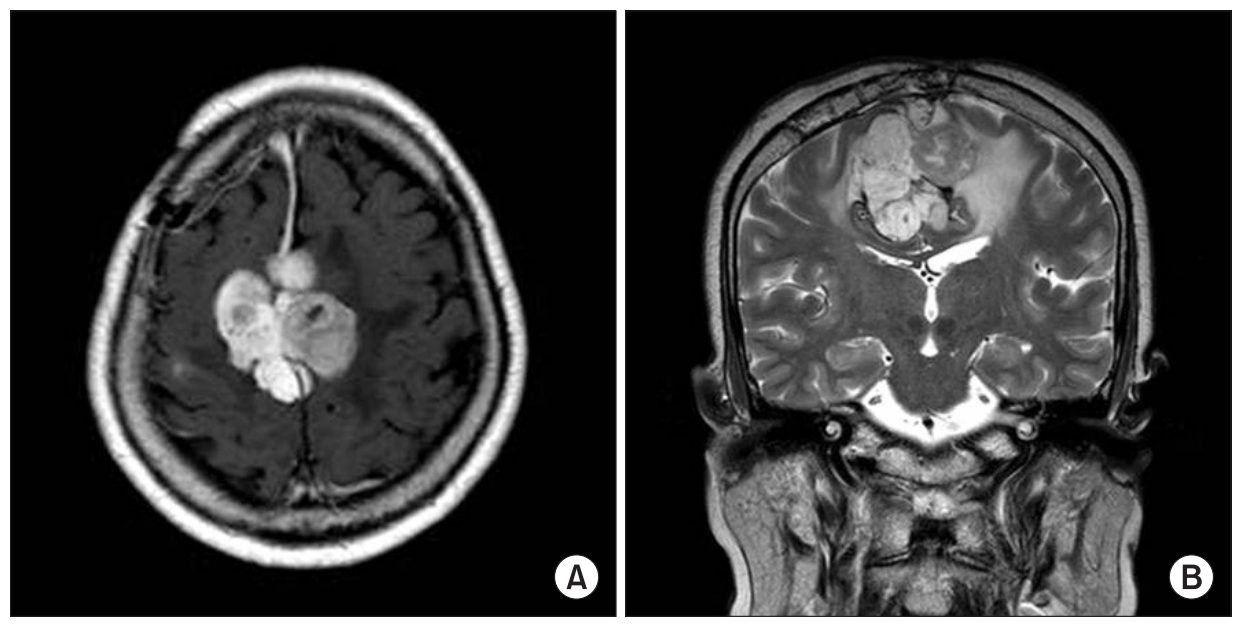

Fig. 1. Enhanced brain magnetic resonance imaging of patient (case 1): (A) coronal view and (B) sagittal view.
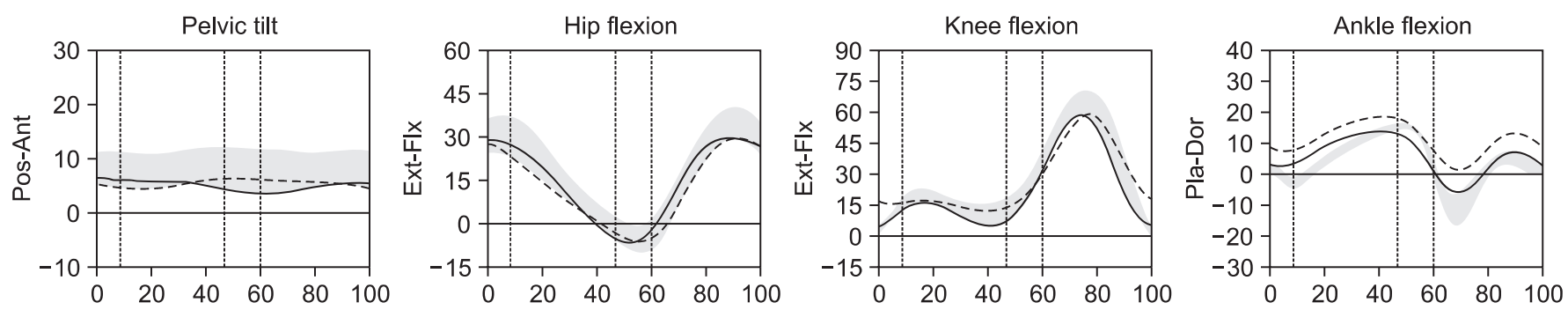

Fig. 2. Gait analysis of patient 1 (a solid line, right side; a dotted line, left side). Pos, posterior; Ant, anterior; Ext, extention; Flx, flexion; Pla, planta flexion; Dor, dorsiflexion.

\section{CASE REPORTS}

\section{Case 1}

In May 2008, a 60-year-old woman developed paraparesis and was diagnosed with falx meningioma (Fig. 1). On June 23, 2008, she underwent a tumor resection surgery and on July 1,2008 , she was transferred to the department of rehabilitation medicine for rehabilitation of paraparesis.

On July 1, 2008, neurologic examination showed grade I spasticity on the Modified Ashworth Scale (MAS) at hip, knee, and ankle joints; a hip flexor (right/left) power of fair grade/fair(-) grade, a hip extensor power of poor grade/ trace grade, a knee extensor power of fair(-) grade/poor(+) grade, a knee flexor power of fair grade/fair(-) grade, an ankle dorsiflexor power of zero grade/zero grade, and an ankle plantar flexor power of zero grade/zero grade. On sensory function tests, she was bilaterally normal. Deep tendon reflexes of the knees and ankles were increased bilaterally and Babinski sign was positive bilaterally. Her lower extremity Fugl-Meyer score was 31/30. After receiv- ing gait training for 2 weeks, she could walk with supervision. Although she did not drag her feet when walking, the motor power of her ankle dorsiflexor was still zero grade. On July 15, 2008, a gait analysis was performed and showed a normal pattern (Fig. 2).

\section{Case 2}

In March 2008, a 48-year-old woman who developed left hemiparesis was diagnosed with a 2-cm-sized low grade glioma in the right superior frontal gyrus. She underwent tumor resection on June 24, 2008 (Fig. 3) and was later transferred to the department of rehabilitation medicine.

On July 19, 2008, neurologic examination showed that the degree of spasticity was MAS grade I at hip, knee, and ankle joints. The power of her left upper extremities was fair grade, and her hip girdle, knee, and ankle powers were trace, zero, and zero grades, respectively. She was normal on sensory function tests. The deep tendon reflexes of her left knee and ankle were increased, and Babinski sign was positive. 

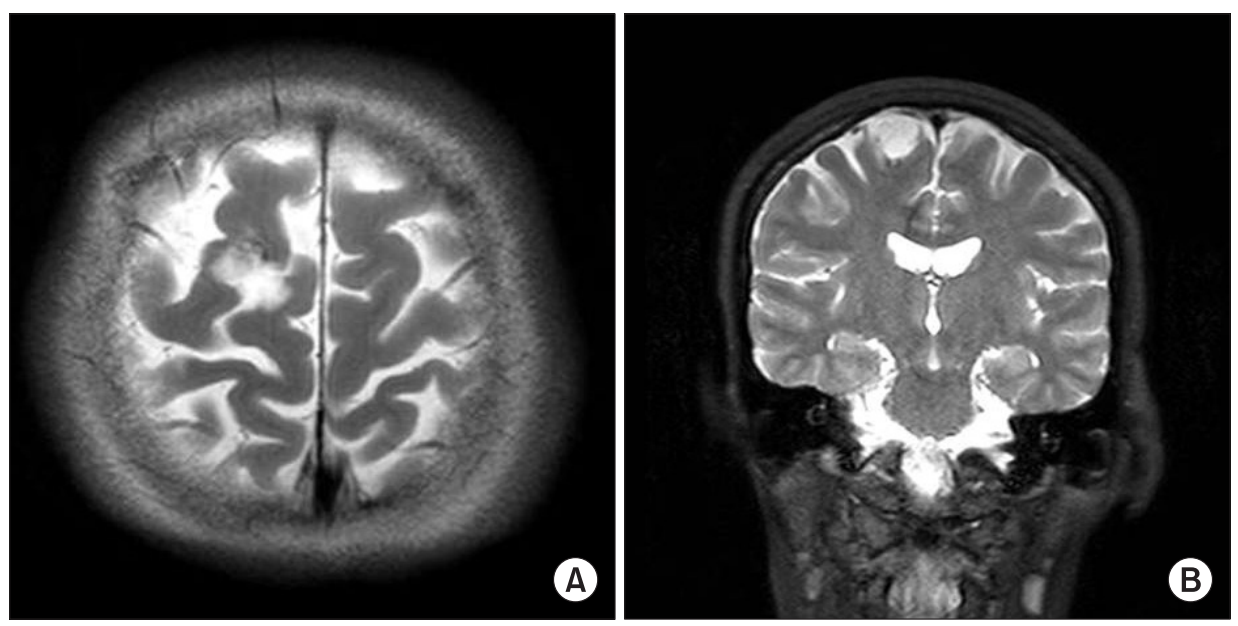

Fig. 3. T2-weighted brain magnetic resonance imaging of patient 2: (A) coronal view and (B) sagittal view.
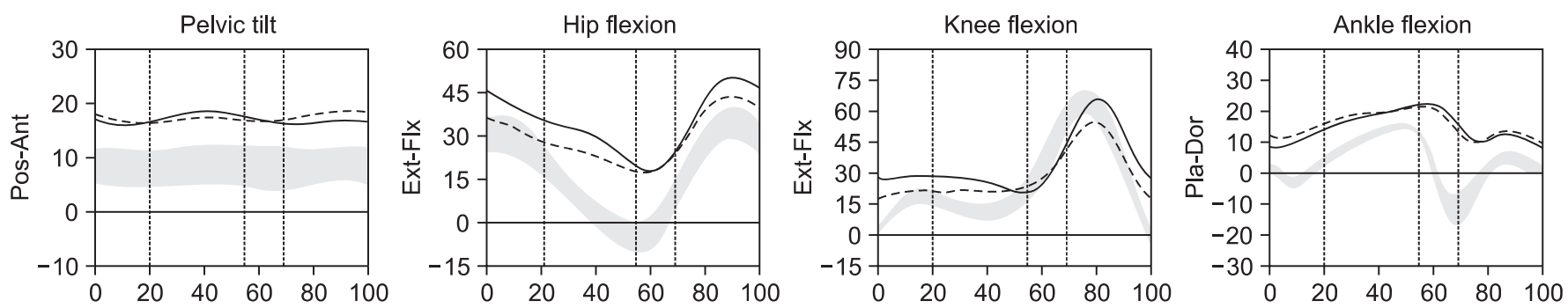

Fig. 4. Gait analysis of patient 2 (a solid line, right side; a dotted line, left side). Pos, posterior; Ant, anterior; Ext, extention; Flx, flexion; Pla, planta flexion; Dor, dorsiflexion.

After receiving rehabilitation treatment for 2 weeks, her motor function was much improved. The power of her left upper extremity was good grade, her hip flexor power fair(+) grade, her extensor power poor grade, her knee flexor power fair(+) grade, her extensor power poor grade, her ankle dorsiflexor power trace grade, her plantar flexor power poor grade, and her lower extremity Fugl-Meyer score 17. Her gait function was much improved, and she was able to walk independently. Though her ankle dorsiflexor was still trace grade, she did not show foot-dragging during the swing phase. On August 2, 2008, a gait analysis was performed, which showed a decreased range of motion at ankle plantarflexion and dorsiflexion. But ankle dorsiflexion was sufficient not to show foot-dragging. Other findings were not significant (Fig. 4).

\section{DISCUSSION}

Our patients showed common features. The brain tumors were located in the superior frontal gyrus (SMA), and the degree of spasticity in either patient was MAS grade I. Motor weakness developed primarily in the lower extremities, with hip flexor of more than grade fair and ankle dorsiflexor grade of zero to trace. The knee flexor was stronger than the knee extensor. Both patients showed difficulties in the initiation of movements but could walk without deficit. There was dissociation between volitional and automatic movements of the affected limbs (Figs. 2, 4).

SMA is important in initially programming and subsequently initiating complex motor sequences which involve bilateral hand coordination and postural preparation for distal extremity movement [5]. SMA lesions characteristically cause a clinical syndrome that evolves over three stages: 1) global akinesia that is worse contralaterally; 2) a sudden recovery a few days later, but with a persistent reduction in contralateral motor activity; and 3) subtle sequelae within weeks to months after surgery [5]. Clinical findings have been confirmed by other series of surgical resection of the SMA $[5,6]$, and the automatic movements in our patients were confirmed by gait analy- 
sis.

Complete SMA syndrome was defined as contralateral hemiplegia with or without mutism, and partial SMA syndrome was classified as contralateral hemiparesis and/ or speech hesitancy [5]. In a previous study which evaluated 27 patients at postoperative 1 day, complete SMA syndrome was developed in 3 patients (11\%) and partial SMA syndrome in 4 (15\%). At postoperative 1 week, complete SMA syndrome was developed in 1 patient (4\%) and partial SMA syndrome in 2 (7.5\%) [5]. In another study which evaluated 6 patients, one patient demonstrated speech abnormality in the form of difficulties in the initiation and halting of speech [7].

Our patients did not show speech hesitancy or mutism. The characteristic features of motor and speech deficits in SMA syndrome seem to be dependent on the tumor location and size of tumor resection $[5,7]$.

A previous study has reported that residual activity was present in an oscillatory neural loop of the thalamocortical and corticocortical networks. A short-term plasticity mechanism based on rapid unmasking of parallel networks was advanced as a hypothesis to explain the phenomenon [8]. Our hypothesis is rather that the development of reflex pattern may contribute to the generation of automatic movements seen. We attempted to determine how our patients activated the ankle dorsiflexor during the swing-phase of the gait cycle. This cycle consists of a stance and a swing phase, and completion of the stance phase is followed by a pre-swing and an initial swing phase. During the latter two phases, rapid hip flexion of up to $40^{\circ}$ is required, and knee flexion may be up to $60^{\circ}$. Such flexion movement may induce dorsiflexion of the ankle (flexor synergy of the ankle).

There is a growing body of evidence that altered reflex pathways may contribute to the abnormal patterns of activation, or muscle synergy. Cortical injury has been shown to result in reorganization of neural connections, leading to changes in the strength of reflex excitability and exerting a substantial influence on spinal networks [9]. The identified patterns of stretch reflex coordination have been found to be consistent with stereotypical voluntary flexion synergy, suggesting that reflex pathways contribute to abnormal muscle coordination $[3,10]$.

The lesions of our patients were located in the medial frontal gyrus and falx, areas from which the anterior corticospinal tract originates. These lesional locations might show significant correlation with the flexor synergy of lower extremities.

We report two unusual cases of flexor synergy after tumor resection of SMA lesions. It is important to recognize SMA syndrome and differentiate it from motor weakness which occurs following primary motor cortical damage, as the former is generally transient and has a good prognosis for a complete recovery.

\section{CONFLICT OF INTEREST}

No potential conflict of interest relevant to this article was reported.

\section{REFERENCES}

1. Penfield W, Welch K. The supplementary motor area of the cerebral cortex; a clinical and experimental study. AMA Arch Neurol Psychiatry 1951;66:289-317.

2. Dewald JP, Beer RF. Abnormal joint torque patterns in the paretic upper limb of subjects with hemiparesis. Muscle Nerve 2001;24:273-83.

3. Trumbower RD, Ravichandran VJ, Krutky MA, Perreault EJ. Altered multijoint reflex coordination is indicative of motor impairment level following stroke. Conf Proc IEEE Eng Med Biol Soc 2008;2008:3558-61.

4. Aruin AS. Simple lower extremity two-joint synergy. Percept Mot Skills 2001;92:563-8.

5. Russell SM, Kelly PJ. Incidence and clinical evolution of postoperative deficits after volumetric stereotactic resection of glial neoplasms involving the supplementary motor area. Neurosurgery 2007;61(1 Suppl):358-67.

6. Fontaine D, Capelle L, Duffau H. Somatotopy of the supplementary motor area: evidence from correlation of the extent of surgical resection with the clinical patterns of deficit. Neurosurgery 2002;50:297-303.

7. Bannur U, Rajshekhar V. Postoperative supplementary motor area syndrome: clinical features and outcome. Br J Neurosurg 2000;14:204-10.

8. Duffau H, Lopes M, Denvil D, Capelle L. Delayed onset of the supplementary motor area syndrome after surgical resection of the mesial frontal lobe: a time course study using intraoperative mapping in an awake patient. Stereotact Funct Neurosurg 2001;76:7482. 
9. Nudo RJ, Plautz EJ, Frost SB. Role of adaptive plasticity in recovery of function after damage to motor cortex. Muscle Nerve 2001;24:1000-19.

10. Thilmann AF, Fellows SJ, Garms E. The mechanism of spastic muscle hypertonus: variation in reflex gain over the time course of spasticity. Brain 1991;114:23344. 